\title{
Remark on the Secular Change in the Energy Density Spectrum of the Geomagnetic Field
}

\author{
Joachim MEYER \\ Institut für Geophysik der Universität Göttingen, Herzberger Landstr. 180, \\ 3400 Göttingen, Federal Republic of Germany
}

(Received July 9, 1985; Revised September 9, 1985)

In two interesting studies of geomagnetic secular variation (S.V.) models AllDREDGE (1984a, b) has evaluated the coefficients of a spherical harmonic expansion of the main field and their first time derivative independently with the spatial spectrum method. By comparing both spectra he concludes that the higher harmonics are more important in the description of S.V. than they are in the description of the main field, a result which he himself considers unfortunate. In fact, if his conclusion were true, it would in principle render further investigations of S.V. more difficult, as much higher degrees would have to be involved in order to gain the same relative accuracy. A consequence of such significance certainly justifies a critical examination of the formal arguments on which the conclusion is based.

To determine the relative importance of each degree to the main field, Alldredge (1984a, b) uses the function

$$
R_{n}=(n+1) \sum_{m=0}^{n}\left[\left(g_{n}^{m}\right)^{2}+\left(h_{n}^{m}\right)^{2}\right]
$$

composed of the summed squares of the Gauss coefficients $g_{n}^{m}, h_{n}^{m}$ of the same degree $n$. It was first derived by MAUERSBERGER (1956) and LUCKE (1957) in two different ways, but is now mostly assigned to LowES (1966). Physically, $R_{n}$ expresses the mean-square value over the Earth's surface of the field produced by all harmonics of the specific degree $n$. Thus $R_{n}$ measures the mean energy density of that particular multipole field constituent at the Earth's surface, except for a factor of $1 / 2 \mu_{0}$ ( $\mu_{0}$ being the permeability of vacuum). It is, therefore, often called the spatial spectrum of the field.

For a corresponding evaluation of the S.V. field Alldredge (1984a, b) then uses the same expression with dots over $g_{n}^{m}, h_{n}^{m}$ and $R_{n}$ to designate the time derivative:

$$
\dot{R}_{n}^{*}=(n+1) \sum_{m=0}^{n}\left[\left(\dot{g}_{n}^{m}\right)^{2}+\left(\dot{h}_{n}^{m}\right)^{2}\right]
$$

where the asterisk has been added here in order to distinguish the function in (2) from the true time derivative of $R_{n}$ given below. By analogy to the interpretation of $R_{n}$ in (1), $\dot{R}_{n}^{*}$ expresses the mean-square value over the Earth's surface 
of the rate of change of the field, i.e., the mean-square of a difference vector (within one year), produced by all harmonics of degree $n$. It is a quantity without any physical meaning in terms of energy density. In particular, it does not express the rate of change of the mean-square value of the field itself which can only be obtained by really differentiating Eq. (1). As is readily apparent, it gives

$$
\dot{R}_{n}=2(n+1) \sum_{m=0}^{n}\left[g_{n}^{m} \dot{g}_{n}^{m}+h_{n}^{m} \dot{h}_{n}^{m}\right]
$$

Physically, $\dot{R}_{n}$ measures the rate of change, i.e., the S.V. of the mean energy density of the particular multipole field constituent at the Earth's surface, again except for a factor of $1 / 2 \mu_{0}$. Thus it is the function $\dot{R}_{n}$ which may be called the spatial spectrum of S.V.

Figure 1 shows $R_{n}$ in curve (a) and $\dot{R}_{n}$ in curve (b) versus $n$ as computed from the IGRF 1980 (PEDDIE, 1982). Whereas curve (a) for $R_{n}$ is exactly the same as the equally labelled curve in Fig. 5 of the paper by ALLDREDGE (1984b), curve (b) for $\dot{R}_{n}$ shows a considerable change as compared to Alldredge's curve for $\dot{R}_{n}^{*}$. Both magnitude and average slope are essentially different. The sign can be either positive (encircled dots) or negative (encircled crosses), but it is only the magnitude irrespective of sign which need be considered in examining the relative importance of each degree.

In comparing the average slope of the two curves in Fig. 1, the following qualifications must be taken into account. Firstly, in the spatial spectrum of the field, i.e., in curve (a), the dipole part $(n=1)$ clearly stands above the general

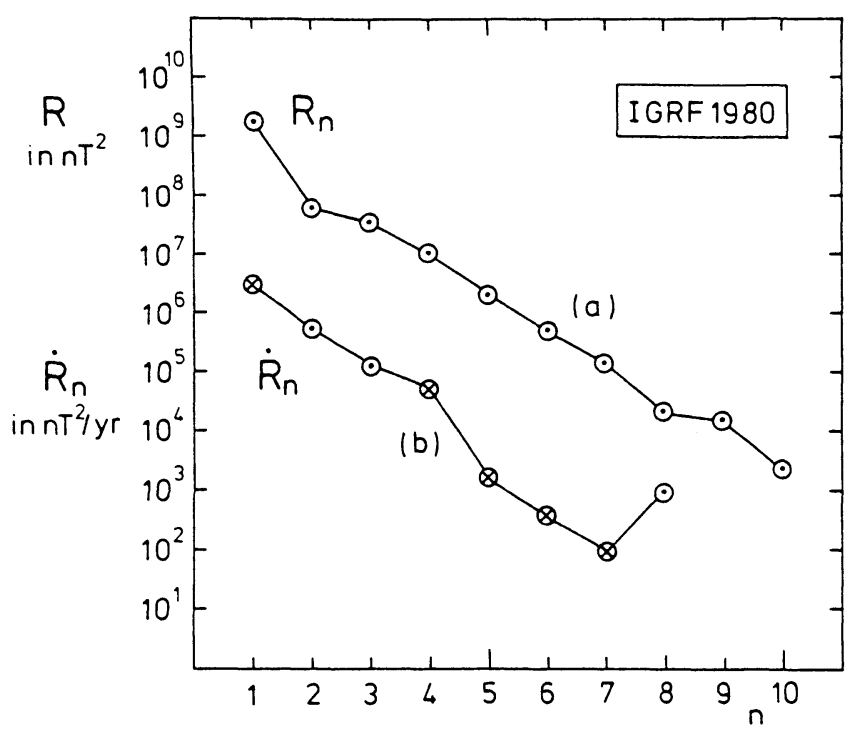

Fig. 1. Contribution of each harmonic degree to the mean square value of the field over the surface of the Earth (curve a) and its first time derivative (curve b), both for the IGRF 1980. 
trend of the higher degree terms, requiring obviously a separate treatment (MEYER et al., 1983). Secondly, in the spatial spectrum of the S.V., i.e., curve (b) the accuracy of the single terms is rapidly decreasing with increasing $n$ because of the different relative weights of the original field models involved in the IGRF coefficients $g_{n}^{m}, h_{n}^{m}$ and their time derivative $\dot{g}_{n}^{m}, \dot{h}_{n}^{m}$ (PEDDIE, 1982) which both contribute to $\dot{R}_{n}$, according to Eq. (3). Deviations among the field models involved are certainly getting more important as the magnitude of the coefficients decreases. In view of these qualifications it can be noticed that the slope of curve (b) from $n=1$ to 4 is very nearly the same as the average slope of curve (a) from $n=2$ to 10 .

The accuracy of the spatial spectrum of S.V. can be improved, if a single one of the basic field models used to derive the IGRF 1980 is evaluated in the same way. Figure 2 shows the result for the model USGS 1980 (PEDDIE and FABIANO, 1982). The improvement is clearly visible, especially regarding the lower part of $\dot{R}_{n}$. The two straight lines are the regression lines computed for both spectra, disregarding only the dipole term for $R_{n}$. The tangent of their respective slope angle is -0.58 for $R_{n}$ and -0.62 for $\dot{R}_{n}$. In consideration of a mean error of \pm 0.06 for the latter, both values are in good accordance.

Figure 3 shows the result for another field model (U041580) developed by J.C. Cain (personal communication). It is based on pre-Magsat data from 1957-1978 and refers to the epoch of 1965.0. Although the model itself includes coefficients up to $n=24$, only the main field part of the spatial spectrum, up to $n=12$, has been evaluated here $\left(R_{n}\right)$. As to the spectrum of S.V. $\left(\dot{R}_{n}\right)$, there

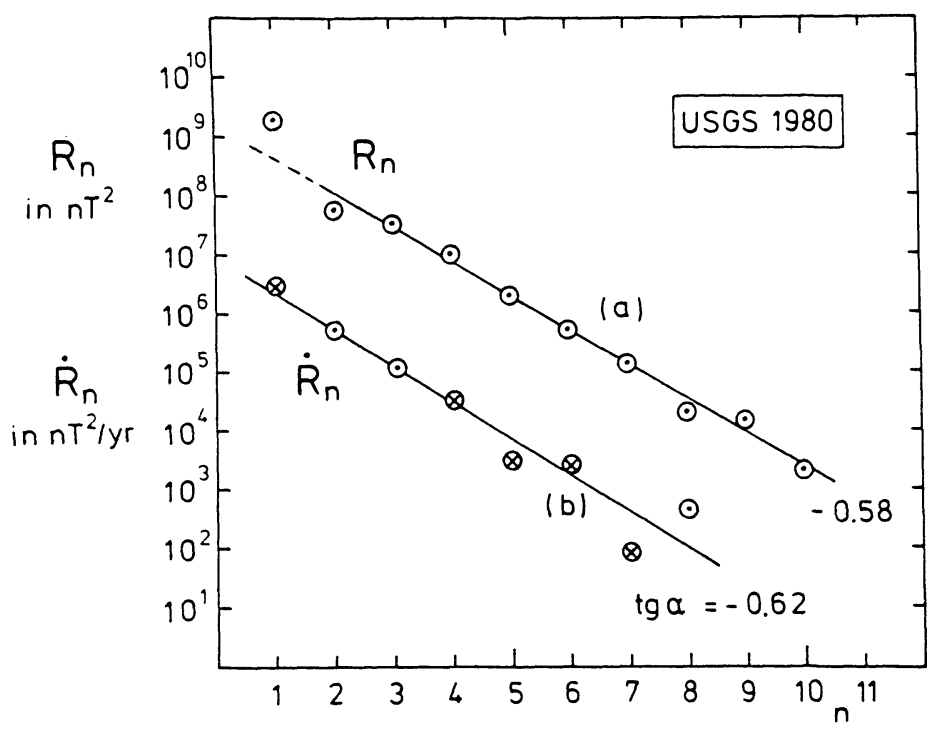

Fig. 2. Same as Fig. 1, but for the field model USGS 1980. The tangent of the slope angle $\alpha$ is indicated for both regression lines. 


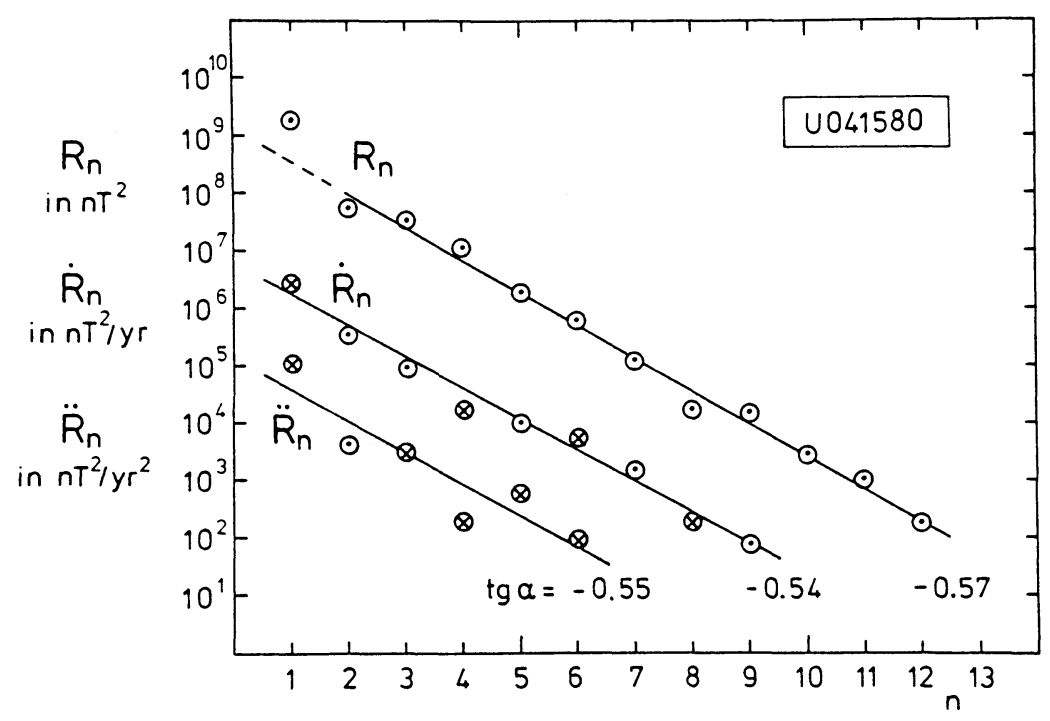

Fig. 3. $R_{n}$ and $\dot{R}_{n}$ same as in Fig. 1 and 2, but for Cain's field model U041580. Shown also is the second time derivative $\ddot{R}_{n}$. Indicated for each regression line is the tangent of the slope angle $\alpha$.

is sufficient accuracy for the time derivatives of the coefficients to include terms up to $n=9$. In addition, the lowest diagram in Fig. 3 shows the second time derivative $\ddot{R}_{n}$ of the magnetic mean energy density at the Earth's surface up to the degree of 6 . It has been computed from the first and second time derivatives of the Gauss coefficients including the coefficients themselves, according to

$$
\ddot{R}_{n}=2(n+1) \sum_{m=0}^{n}\left[g_{n}^{m} \ddot{g}_{n}^{m}+h_{n}^{m} \ddot{h}_{n}^{m}+\left(\dot{g}_{n}^{m}\right)^{2}+\left(h_{n}^{m}\right)^{2}\right] .
$$

Despite an increased scatter of the different terms, the magnitude of the single harmonics again reveals a superimposed, quasi linear dropoff with obviously the same slope as appears for $R_{n}$ and $\dot{R}_{n}$. In fact, the slope of the regression line, given in Fig. 3 as tangent of the slope angle $\alpha$, is the same in all three cases, within the limits of statistical error. It has already been shown that its specific value indicates the depth of the effective source layer (MEYER et al., 1983). Accordingly, the relative importance of the higher harmonics in the spectrum of S.V. as defined above appears to be absolutely the same as it is in the spectrum of the main field itself. Alldredge's diverging conclusion results from using a different formula.

In this connexion mention must further be made of the predicted crustal part in the spatial spectrum of S.V., as inferred from a global model of crustal susceptibility distribution and different inducing core fields (MEYER et al., 1985). 
The complete spectrum of S.V. to be observed at the Earth's surface has very likely a similar shape as the spatial spectrum of the internal field, consisting of a linearly decreasing core field part and a quasi-white part of crustal origin.

\section{REFERENCES}

AlldRedge, L. R., Harmonics required in main field and secular variation models, J. Geomag. Geoelectr., 36, 63-72, 1984a.

AlldREDGE, L. R., Geomagnetic secular variation and varying dipoles in the core, J. Geomag. Geoelectr., 36, 621-633, 1984b.

LOWES, F. J., Mean-square values on sphere of spherical harmonic vector fields, J. Geophys. Res., 71, $2179,1966$.

LuCKE, O., Über Mittelwerte von Energiedichten der Kraftfelder, Wiss. Z. Päd. Hochschule Potsdam, Math.-Nat. Reihe, 3, 39-46, 1957.

Mauersberger, P., Das Mittel der Energiedichte des geomagnetischen Hauptfeldes an der Erdoberfläche und seine säkulare Änderung, Gerlands Beitr. Geophys., 65, 207-215, 1956.

Meyer, J., J. - H. Hufen, M. SieBert, and A. HAHN, Investigations of the internal geomagnetic field by means of a global model of the Earth's crust, J. Geophys., 52, 71-84, 1983.

Meyer, J., J.-H. HufEN, M. Siebert, and A. HAHN, Ein Krustenanteil im räumlichen Spektrum der erdmagnetischen Säkularvariation, J. Geomag. Geoelectr., 37, 1985 (in press).

PEDDIE, N. W., International Geomagnetic Reference Field: the third generation, J. Geomag. Geoelectr., 34, 309-326, 1982.

Peddie, N. W. and E. B. FABIANO, A proposed International Geomagnetic Reference Field for 1965-1985, J. Geomag. Geoelectr., 34, 357-364, 1982. 